\title{
Bundled care in acute kidney injury in critically ill patients, a before-after educational intervention study
}

Jacqueline Koeze* ${ }^{*}$, Iwan C. C. van der Horst, Renske Wiersema, Frederik Keus, Willem Dieperink, Eline G. M. Cox, Jan G. Zijlstra and Matijs van Meurs

\begin{abstract}
Background: Acute kidney injury (AKI) often occurs in critically ill patients. AKI is associated with mortality and morbidity. Interventions focusing on the reduction of AKI are suggested by the Kidney Disease: Improving Global Outcomes guideline. We hypothesized that these educational interventions would improve outcome in patients admitted to the Intensive Care Unit (ICU).

Methods: This was a pragmatic single-centre prospective observational before-after study design in an ICU in a tertiary referral hospital. All consecutive patients admitted to the ICU irrespective their illness were included. A 'Save the Kidney' (STK) bundle was encouraged via an educational intervention targeting health care providers. The educational STK bundle consisted of optimizing the fluid balance (based on urine output, serum lactate levels and/ or central venous oxygen saturation), discontinuation of diuretics, maintaining a mean arterial pressure of at least $65 \mathrm{mmHg}$ with the potential use of vasopressors and critical evaluation of the indication and dose of nephrotoxic drugs. The primary outcome was the composite of mortality, renal replacement therapy (RRT), and progression of AKI. Secondary outcomes were the components of the composite outcome the severity of AKI, ICU length of stay and in-hospital mortality.
\end{abstract}

Main results: The primary outcome occurred in 451 patients (33\%) in the STK group versus 375 patients (29\%) in the usual care group, relative risk (RR) 1.16, 95\% confidence interval (CI) 1.03-1.3, $p<0.001$. Secondary outcomes were, ICU mortality in $6.8 \%$ versus $5.6 \%$, (RR $1.22,95 \% \mathrm{Cl} 0.90-1.64, p=0.068$ ), RRT in $1.6 \%$ versus $3.6 \%$ (RR $0.46,95 \%$ $\mathrm{Cl} 0.28-0.76, p=0.002$ ), and AKI progression in $28 \%$ versus $24 \%$ (RR $1.18,95 \% \mathrm{Cl} 1.04-1.35, p=0.001$ ).

Conclusions: Providing education to uniformly apply an AKI care bundle, without measurement of the implementation in a non-selected ICU population, targeted at prevention of AKI progression was not beneficial.

Keywords: AKI prevention, ICU, Care-bundle

\footnotetext{
* Correspondence: j.koeze@umcg.nl

Department of Critical Care, University of Groningen, University Medical

Center Groningen, Postbus 30.001, 9700, RB, Groningen, The Netherlands
}

(c) The Author(s). 2020 Open Access This article is licensed under a Creative Commons Attribution 4.0 International License, which permits use, sharing, adaptation, distribution and reproduction in any medium or format, as long as you give appropriate credit to the original author(s) and the source, provide a link to the Creative Commons licence, and indicate if changes were made. The images or other third party material in this article are included in the article's Creative Commons licence, unless indicated otherwise in a credit line to the material. If material is not included in the article's Creative Commons licence and your intended use is not permitted by statutory regulation or exceeds the permitted use, you will need to obtain permission directly from the copyright holder. To view a copy of this licence, visit http://creativecommons.org/licenses/by/4.0/ The Creative Commons Public Domain Dedication waiver (http://creativecommons.org/publicdomain/zero/1.0/) applies to the data made available in this article, unless otherwise stated in a credit line to the data. 


\section{Background}

Acute kidney injury (AKI) occurs in 15 to $33 \%$ of all critically ill patients $[1,2]$. The initial definition of AKI was defined as an increase in serum creatinine and/or a reduction in urine output by Bellomo et al. in 2004, which was revised by Mehta et al. in 2007 and once again in the current definition by Kidney Disease: Improving Global Outcomes (KDIGO) in 2012 [3-5]. Irrespective of the definition, the occurrence of AKI is associated with increased mortality rates and also increased incidences of chronic kidney disease (CKD) after discharge $[6,7]$. The severity of AKI is associated with increased intensive care unit (ICU) and hospital mortality [8].

Despite the lack of evidence for benefit, guidelines directed to prevent AKI recommend either volume resuscitation or volume restriction, promote and dismiss various types of fluids, pose blood pressure targets, and emphasize avoidance of nephrotoxic drugs [5]. The recommendation of controlled fluid resuscitation is based on observations that both hypovolemia and hypervolemia can induce AKI [9]. The use of artificial colloids and (loop-) diuretics is dissuaded based on aggregated data from randomised trials and large cohort studies $[5,10]$. Nephrotoxic drugs and drug dosing should be weighed carefully, especially in critically ill patients already at risk for AKI [5]. Further, guidelines recommend targeting blood pressure at a mean arterial pressure (MAP) above 60-65 mmHg using vasopressors $[5,11]$.

A bundled approach of care for the critically ill including these individual recommendations is suggested to preserve renal function [5]. Care bundles targeted at the treatment of sepsis and ventilator-associated pneumonias (VAP) have improved outcomes in critically ill patients [12-14]. Bundles targeted at AKI prevention have already been successfully tested in patients undergoing cardiac surgery and also in hospitalized patients, but not in a general critically ill population $[15,16]$.

We therefore hypothesized that the implementation of education for bundled care targeted at prevention of AKI progression and a reduction in AKI severity, would improve patient outcome.

\section{Methods}

\section{Study design}

A pragmatic single-centre prospective observational before-after study design was used. The study was performed in a tertiary referral hospital. The need for informed consent was waived by the Institutional Review Board of our hospital (METc 2013-174). The Ethical Board waived consent because the care bundle was implemented at a department level and changed overall daily practice. Patients were treated according to usual care during the first period. After the first ('before') period of the study the educational intervention was introduced at a department level to all personnel between June 12th, 2015 and October 14th, 2015. During the second period patients were treated according to the 'Save the Kidney' (STK)-bundle in addition to usual care. The duration of both study periods was estimated from admittance rates combined with sample size calculations.

\section{Patient selection}

Consecutive patients admitted to the ICU were included irrespective their illness. Both scheduled post-surgery patients needing postoperative ICU observation and acutely admitted patients, both surgical and medical, were included. Patients with known chronic kidney disease (defined by a serum creatinine greater than $177 \mu \mathrm{mol} / \mathrm{l}$, based on the definition used by the Nationale Intensive Care Evaluatie (NICE)) and patients on chronic renal replacement therapy (RRT) were excluded from the analysis. If patients were readmitted to our ICU within the study period only data of their first admission were included in the analysis. Patients were splitted into two groups based on admission date, divided into two set periods of time.

\section{Data collection}

Baseline data of all patients were recorded, including age, sex, body mass index (BMI), APACHE IV and admission category and type (medical or surgical; scheduled or emergency). In addition to age and severity of illness, the presence of known diabetes mellitus was recorded as it is a known risk factor for AKI development. We recorded mortality (ICU and hospital), the need for RRT, the occurrence and severity of AKI and the length of ICU stay. Serum creatinine was recorded each day and urinary output was recorded hourly. The reference creatinine was based on the ideal serum creatinine, which was calculated assuming a clearance of $75 \mathrm{ml} /$ $\mathrm{min} / 1.73 \mathrm{~m}^{2}$ using the 'modification of diet in renal disease' (MDRD) formula. The incidence and severity of AKI were assessed in each patient according to the KDIGO definitions, using both serum and urine output criteria (Supplementary figure 1). AKI progression was defined as any progression (i.e. 0 to 1,2 to 3, etc.) in AKI stage during the first $48 \mathrm{~h}$ of admission.

\section{The 'save the kidney' educational intervention bundle}

The STK bundle was encouraged in all patients (Table 1). The STK bundle consisted of optimizing the fluid balance (based on urine output, serum lactate levels and/or central venous oxygen saturation), discontinuation of diuretics, maintaining a MAP of at least $65 \mathrm{mmHg}$ with the potential use of vasopressors and critical evaluation of the indication and dose of nephrotoxic drugs [5]. Discouraging artificial colloids was not part of the bundle, since colloids were already excluded in the past (Table 1). Avoidance of hyperglycaemia as is suggested by the KDIGO guidelines 
Table 1 The 'Save the Kidney'-bundle

Optimize fluid balance
CVP $8-12 \mathrm{mmHg}$

UO $>0.5 \mathrm{ml} / \mathrm{kg} / \mathrm{h}$

serum lactate $<2 \mathrm{mmol} /$

$\mathrm{SvO} 2>65 \%$

Stop diuretics

MAP $>65 \mathrm{mmHg}$

Evaluate the indication and dose of possible nephrotoxic medication

CVP Central venous pressure, UO Urinary output, SVO2 Mixed venous oxygen saturation, MAP Mean arterial pressure

was not part of the bundle, as we already used a computerised algorithm regulating serum glucose which was the same in both groups [17]. Close monitoring of serum creatinine, also suggested by the KDIGO guidelines, was also the same in both groups. As mentioned before we measured serum creatinine daily and urine output hourly.

The 'STK'-bundle was introduced using introductory lectures to all physicians and nurses, by the distribution of pocket cards, and emphasized during discussions on the wards. The specific interventions were left open for the treating physician to make the bundle more compliant with different personal preferences within the treating intensivist group. We did not assess compliance with the bundle.

\section{Outcomes}

The primary outcome measure was a composite of serious adverse events (SAE's) consisting of ICU mortality, the need for RRT and/or the progression of AKI.

Secondary outcomes were the three individual components of the composite primary outcome, including ICU mortality, the need for RRT, and the progression of AKI during the first $48 \mathrm{~h}$ of ICU stay. In-hospital mortality and ICU length of stay were considered as well.

\section{Sample size estimation}

A previous study in a comparable critically ill population observed a 30 -day mortality of $17 \%$, a cumulative incidence proportion of approximately $5 \%$ for RRT and the cumulative incidence proportion of $16 \%$ for AKI [1]. Based on these data, we estimated a cumulative risk of $25 \%$ of one or more of the SAE in the control group of our ICU population. With a cumulative event rate of $25 \%$ in the control group, a relative risk reduction (RRR) of $20 \%$ of SAE (which would imply a reduction of SAE from 25 to $20 \%$, which was considered clinically relevant), a type I error of 0.05 , and a type II error of 0.20 , we calculated that 1094 patients were needed in each group of the study (and 2188 patients in total). We anticipated a study period of 6 months for each group.

\section{Statistical analysis}

The proportions of AKI were calculated based on data of the first $48 \mathrm{~h}$ of ICU admission. AKI severity was calculated in all patients. Dichotomous data were presented as percentages. Continuous variables were reported as means (with standard deviations (SD)) or as medians (with interquartile ranges (IQR)) depending on normality. Data were analysed using Student t-tests, Mann Whitney U tests, or Chi-square tests, when appropriate. As a sensitivity analysis, analysis were repeated in patients with and without diabetes mellitus.

Missing hourly urine output data were replaced based on averages using the first recorded value over the missing hours. Urine output data were omitted from the analysis if all hourly urine output was missing.

\section{Results}

A total of 3822 patients were admitted between the start of the study January 1st, 2014 and study closure on March 15th, 2015. The first period was from 01 to 01 2014 until 11-06-2014 and the second period was from 15 to 10-2014 until 15-03-2015. A total of 1295 patients (49\%) were included in the usual care group and 1347 patients (51\%) were included in the STK group. (Fig. 1a).

\section{Baseline patient characteristics}

Patients in both groups were similar regarding age, sex, BMI, severity of illness and the presence of known diabetes mellitus (Table 2). More patients in the STK group were admitted for a medical reason and less patients were admitted after scheduled surgery (Table 2). Patients in the STK group had a lower median cumulative fluid balance during the first 3 days of ICU admission.

Serum creatinine at admission was unavailable in 98 patients (3.7\%) and in 489 patients (19\%) there was insufficient data to estimate the presence or absence of AKI based on urine output criteria on admission. Overall, the median available hours of urine output data calculated as a percentage of length of stay was 89\% (IQR 62-95\%).

\section{Primary outcome}

Serious adverse events defined as ICU mortality, the need for RRT and/or the progression of AKI was observed in 451 patients (33\%) in the STK group compared to 375 patients (29\%) in the usual care group (RR 1.16, 95\% confidence interval CI 1.03-1.3, $p<0.001$ ) (Table 3). 


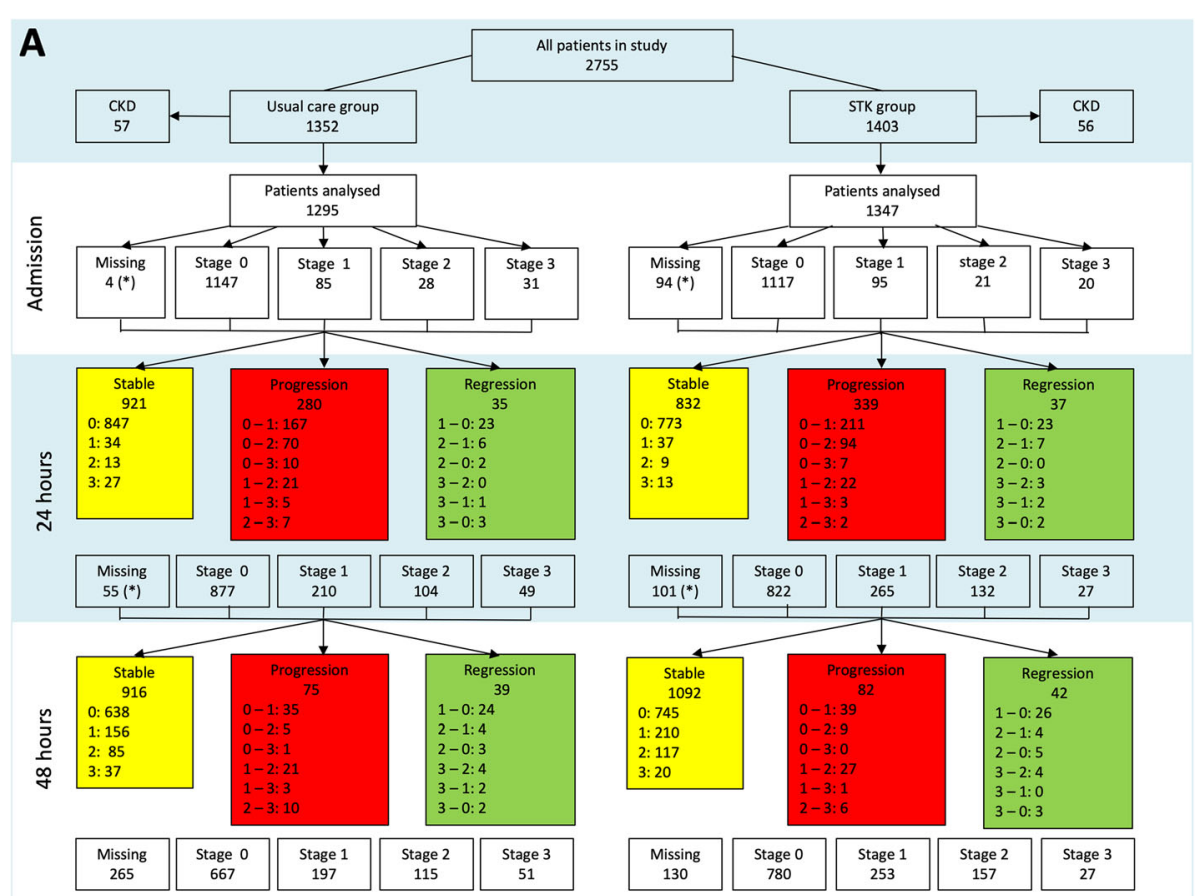

B

\section{Control group}

\begin{tabular}{|c|c|c|c|c|c|c|}
\hline & \multicolumn{6}{|c|}{ Maximum AKI stage during first 48 hours } \\
\hline \multirow{5}{*}{ 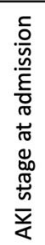 } & & 0 & 1 & 2 & 3 & Total \\
\hline & 0 & 861 & 182 & 89 & 15 & 1147 \\
\hline & 1 & 23 & 31 & 19 & 12 & 85 \\
\hline & 2 & 2 & 7 & 11 & 8 & 28 \\
\hline & 3 & 2 & 0 & 2 & 27 & 31 \\
\hline
\end{tabular}

\section{STK group}

\begin{tabular}{|c|c|c|c|c|c|c|}
\hline & \multicolumn{6}{|c|}{ Maximum AKI stage during first 48 hours } \\
\hline \multirow{5}{*}{ 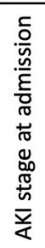 } & & 0 & 1 & 2 & 3 & Total \\
\hline & 0 & 761 & 225 & 119 & 12 & 1117 \\
\hline & 1 & 22 & 42 & 27 & 4 & 95 \\
\hline & 2 & 2 & 5 & 11 & 3 & 21 \\
\hline & 3 & 0 & 2 & 4 & 14 & 20 \\
\hline
\end{tabular}

Fig. 1 Flow diagram of patients in the study. a. Numbers of patients admitted in both groups. Numbers of patients excluded due to presence of chronic kidney disease (CKD). Numbers of patients with severity of acute kidney injury (AKI) and with stable AKI, AKI progression or regression at admission, after $24 \mathrm{~h}$ of admission and after $48 \mathrm{~h}$ of admission. $\left(^{*}\right)$ Usual care group: 4 missing AKI stage at admission, 2 stage 0 after $24 \mathrm{~h}$ and 2 stage 1 after $24 \mathrm{~h}$. Save the kidney group: 94 missing AKI stage at admission, 24 stage 0 at $24 \mathrm{~h}, 8$ stage 1 at $24 \mathrm{~h}, 4$ stage 2 at $24 \mathrm{~h}, 2$ stage 3 at $24 \mathrm{~h}$ and 56 missing AKI stage after $24 \mathrm{~h}$. Usual care group: 55 missing AKl stage at $24 \mathrm{~h}$ all remain missing after $48 \mathrm{~h}$. Save the kidney group: 101 missing AKI stage at $24 \mathrm{~h}, 1$ is stage 0 after $48 \mathrm{~h}, 100$ are missing AKI stage at $48 \mathrm{~h}$. b. Number of patients in the control group and STK group with severity of AKI at admission and development over the first $48 \mathrm{~h}$. Green squares indicate improvement in AKI during the first $48 \mathrm{~h}$. Yellow squares indicate stable AKI during the first $48 \mathrm{~h}$. Red squares indicate AKI progression during the first $48 \mathrm{~h}$ 
Table 2 Basic patient characteristics

\begin{tabular}{|c|c|c|c|}
\hline Characteristic & Usual care group & STK group & $P$-value \\
\hline Number of patients & 1295 & 1347 & \\
\hline Age (years; mean $\pm S D$ ) & $60.2(15.6)$ & $59.6(16.2)$ & 0.35 \\
\hline Male sex (N (\%)) & 809 (63) & $835(62)$ & 0.8 \\
\hline Body Mass Index (kg/m2; mean \pm SD) & $26.5(5.2)$ & $26.5(10)$ & 0.98 \\
\hline APACHE IV (mean \pm SD) & $51(25)$ & $52.3(25)$ & 0.18 \\
\hline Admission type & & & 0.05 \\
\hline - Medical (N (\%)) & $423(33)$ & $497(37)$ & \\
\hline \multicolumn{4}{|l|}{ - Surgical (N (\%)) } \\
\hline ○ Scheduled (N (\%)) & $699(54)$ & $665(49)$ & \\
\hline o Emergency (N (\%)) & $172(13)$ & $185(14)$ & \\
\hline \multicolumn{4}{|l|}{ Co-morbidity } \\
\hline - Diabetes mellitus (N (\%)) & $194(15)$ & $195(15)$ & 0.72 \\
\hline Serum creatinine at admission ( $\mu$ mol/l; median (IQR)) & $73(59-91)$ & $72(59-89)$ & 0.16 \\
\hline KDIGO stage admission (N (\%)) & $1291(99)$ & $1253(93)$ & 0.73 \\
\hline No AKI (N (\%)) & $1147(89)$ & $1117(83)$ & \\
\hline Stage 1 (N (\%)) & $85(6.6)$ & $95(7.1)$ & \\
\hline Stage 2 (N (\%)) & $28(2.2)$ & $21(1.6)$ & \\
\hline Stage 3 (N (\%)) & $31(2.4)$ & $20(1.5)$ & \\
\hline Fluid balance day 1 & $1086(299,2112)$ & $728(61,1795)$ & $<0.01$ \\
\hline Fluid balance day 2 & $848(194,2260)$ & $726(173,1878)$ & 0.023 \\
\hline Fluid balance day 3 & $708(89,1785)$ & $533(-13,1389)$ & 0.011 \\
\hline
\end{tabular}

STK 'Save the Kidney', SD Standard deviation, APACHE Acute physiology and chronic health evaluation, IQR Inter quartile range

Table 3 Primary and secondary outcomes of the 'Save the Kidney'-bundle

\begin{tabular}{|c|c|c|c|}
\hline Outcome & Usual care group & STK group & RR $(95 \%$ Cl) $P$-value \\
\hline Number of patients & 1295 & 1347 & \\
\hline \multirow[t]{2}{*}{ Primary outcome (AKI progression, need for RRT and ICU mortality) (N (\%)) } & $388(30)$ & $465(36)$ & $1.15(1.03-1.29)$ \\
\hline & & & $0.001\left(^{*}\right)$ \\
\hline \multirow[t]{2}{*}{ Overall ICU mortality (N (\%)) } & $72(5.6)$ & $91(6.8)$ & $1.22(0.90-1.64)$ \\
\hline & & & $0.068\left(^{*}\right)$ \\
\hline \multirow[t]{2}{*}{ Need for RRT during ICU admission (N (\%)) } & $46(3.6)$ & $22(1.6)$ & $0.46(0.28-0.76)$ \\
\hline & & & $0.002\left(^{*}\right)$ \\
\hline \multirow[t]{2}{*}{ AKI progression ( $(\%))$} & $326(25)$ & $402(31)$ & $1.19(1.05-1.34)$ \\
\hline & & & $0.001(* *)$ \\
\hline Maximum AKI stage during $48 \mathrm{~h}$ & & & $0.87(0.82-0.93)$ \\
\hline No AKI (N (\%)) & $863(67)$ & $783(61)$ & $\left.<0.001{ }^{(* *}\right)$ \\
\hline AKI stage $1(\mathrm{~N}(\%))$ & $238(18)$ & $299(23)$ & $1.21(1.04-1.41)$ \\
\hline AKI stage $2(\mathrm{~N}(\%))$ & $128(10)$ & $169(13)$ & $1.27(1.02-1.58)$ \\
\hline AKI stage $3(\mathrm{~N}(\%))$ & $66(5.1)$ & $41(3.2)$ & $0.6(0.41-0.88)$ \\
\hline ICU LoS (days; median [IQR]) & $2[2-3]$ & $2[2-3]$ & $0.319\left(^{*}\right)$ \\
\hline Hospital mortality (N (\%)) & $117(9)$ & $137(10.2)$ & $0.248\left(^{*}\right)$ \\
\hline
\end{tabular}

STK 'Save the Kidney', AKI Acute kidney injury, RRT Renal replacement therapy, ICU Intensive care unit, LoS Length of stay, IQR Inter quartile range, $R R$ Relative risk, $\mathrm{CI}$ Confidence interval. $\left(^{*}\right): p$ value considering difference between outcome in the usual care group and the STK group. $\left(^{* *}\right) p$ value considering difference in AKI incidence between both groups. $\left.{ }^{* * *}\right) p$ value considering difference in AKI severity between both groups 
The AKI progression during the first $48 \mathrm{~h}$ after ICU admission was not different between the STK group and the usual care group (Table 3 and Fig. 1b).

\section{Secondary outcomes ICU mortality}

In the STK group 91 patients $(6.8 \%)$ died during their ICU admission compared to 72 patients $(5.6 \%)$ in the usual care group (RR 1.22, 95\% confidence interval $0.90-1.64, p=0.068$ ) (Table 3).

\section{Need for RRT}

RRT was used in 22 patients $(1.6 \%)$ in the STK group versus 46 patients $(3.6 \%)$ in the usual care group RRT (RR 0.46, $95 \%$ confidence interval $0.28-0.76, p=0.002$ ) (Table 3).

\section{AKI progression}

Based on serum creatinine and urine output criteria 383 patients (28\%) in the STK group developed AKI progression versus 311 patients $(24 \%)$ in the usual care group (RR 1.18, 95\% confidence interval 1.04-1.35, $p=0.001$ ).

AKI progression based on the separate components serum creatinine and urine output is shown as supplementary material (supplementary data 1 and supplementary table 1). The sensitivity analysis showed that patients with diabetes mellitus did not show a difference in outcome (supplementary table 2).

\section{AKI severity}

Based on serum creatinine and urine output AKI severity differed in the STK group compared with the usual care group. In the STK group 299 patients (23\%) had stage $1 \mathrm{AKI}$ and in the usual care group 238 patients (18\%). In the STK group 169 patients (13\%) had stage 2 AKI and in the usual care group 128 patients (10\%). In the STK group 41 patients $(3.2 \%)$ had stage 3 AKI and in the usual care group $66(5.1 \%)(p<0.001)$ (Table 3).

AKI severity based on the separate components serum creatinine and urine output is shown in the supplementary table 1 .

\section{ICU length of stay}

Median ICU length of stay in the STK group was 2 days [IQR 2-3] and in the usual care group also 2 days [IQR $2-3](p=0.319)$ (Table 3).

\section{In-hospital mortality}

In the STK group 137 patients (10\%) died during their hospitalisation and in the usual care group 117 patients (9\%) (RR 1.13, 95\% confidence interval 0.89-1.42, $p=$ 0.248) (Table 3).

\section{Discussion}

We used a pragmatic before-after design to test whether a bundled approach targeted at prevention of AKI and a reduction of AKI severity and AKI progression, introduced at a department level impacts the outcome of critically ill patients. This study showed that implementation of an educational 'Save the Kidney bundle care in critically ill patients aiming at a reduction of AKI had no beneficial effect on patient outcome when evaluated by a composite of ICU mortality, the need for RRT and AKI progression.

These results are in contrast with the few studies that evaluated AKI care bundles in hospitalized patients in general or in patients after cardiac surgery $[15,16]$. Those studies showed either a reduction in AKI incidence and AKI severity in cardiac surgery patients or a reduction in AKI incidence and a reduction in-hospital mortality. Our study was not powered to detect significance in individual components of the primary endpoint. The primary outcome showed no benefit as a result of a higher AKI incidence and contrary, a lower need for RRT in the STK group (Table 3). In our study ICU mortality was higher in the STK group, albeit nonsignificant. Therewith, the effects of the implemented bundle were contradictory, given the fact that need for RRT is associated with a higher mortality.

Our results are also in contrast with other bundles in critically ill patients such as the treatment bundle of the Surviving Sepsis Campaign (SSC) to reduce mortality in patients with sepsis or the bundle to reduce incidence of ventilator associated pneumonia (VAP) in mechanically ventilated critically ill patients $[13,14]$. It is important to note however that our study comprised an educational intervention of which affects are often difficult to assess.

\section{Strengths and limitations}

Our study has several limitations. First, we did not record which patients had the bundle applied in their treatment. Besides that, we did not record which part of the bundle was applied in which patients. It is possible that one of the suggested interventions of the bundle has detrimental effects on the composite primary outcome, while others may have positive effects. Moreover, not all interventions were clearly described, but adjustments in antibiotic dose were based on renal clearance and accordingly appropriate drug dosing. This also holds for the optimization of the fluid balance, physicians and nurses were stimulated to critically evaluate the need for fluids. This was based on the formulation of the KDIGO guidelines.

Second, we did not assess the full MAKE30 endpoint, which was not published at the time. However, our primary endpoint was the composite of death, new renal replacement therapy, or persistent renal dysfunction which is recommended, as a patient-centered outcome for pragmatic trials involving AKI.

Third, we did not analyse the compliance of the bundle and furthermore, the same bundle can be implemented differently by different clinicians. A recent study showed 
that using an interruptive AKI alert therapeutically interventions in patients increased from 7.9 to $28.7 \%$ of the patients [18]. Therefore, it is likely that the compliance of this study was not $100 \%$. Future research of our group and others on the effect of AKI care bundles in critically ill patients need to address this issue.

Fourth, we did not correct for all previously reported comorbidities, hence, differences in illness severity between groups may have played a role in our findings. Furthermore, we had insufficient urine output data to estimate AKI incidence and severity based on urine criteria in nearly $20 \%$ of patients, with unequal amounts of missing data between the two groups. This might have led to underestimation of AKI incidence in the usual care group since urine output is more sensitive in detecting AKI than serum creatinine. This could have exaggerated the (negative) effect of the care bundle. Last, we did not collect baseline creatinine but estimated renal function based on the assumption of a glomerular filtration rate of $75 \mathrm{l} / \mathrm{min} / 1.73 \mathrm{~m}^{2}$.

A strength of this study is the prospective study design with the inclusion of all patients, except for patients with CKD, which reflects real life daily practice and patient population. Even though this resulted in relatively low median APACHE scores, AKI incidence was still high and the KDIGO guidelines suggest that the measures they advise may reduce AKI incidence in general.

No comparable studies have been performed in critically ill patients. We constructed an educational intervention regarding bundled elements of AKI care as advised by international guidelines. Each of the measures is supported by literature and guidelines suggest beneficial effects of bundled care. The results of our study show a contradictory effect. This may be caused by limitations of the current study or by chance, but it may also be the result of the bundle itself. The simultaneously taken measures rather than measures taken subsequently might induce harm. A possible cause is for example the reduced fluid resuscitation with possible adverse effects on outcome due to - relative - hypovolemia. Also, the reductions in antibiotic dosing propagated by the bundle may have led to insufficient treatment of infections. This may support therapeutic drug monitoring (TDM) [19]. In future research protocol adherence and effects of implementation need to be studied.

\section{Conclusion}

Although more patients in the control group developed stage 3 AKI compared to those in the STK intervention group, providing education to uniformly apply an AKI care bundle, without measurement of the implementation in a non-selected ICU population, targeted at prevention of AKI progression was not beneficial.

\section{Supplementary information}

Supplementary information accompanies this paper at https://doi.org/10. 1186/s12882-020-02029-8.

Additional file 1: Figure S1. AKI classification according to the KDIGO guideline.

Additional file 2. Supplementary data 1.

Additional file 3: Table S1.

Additional file 4: Table S2.

\section{Abbreviations}

AKl: Acute kidney injury; BMI: Body Mass Index; CKD: Chronic Kidney Disease; ICU: Intensive Care Unit; IQR: Inter Quartile Range; KDIGO: Kidney Disease Improving Global Outcome; MAP: Mean Arterial Pressure;

MDRD: Modification of Diet in Renal Disease; NICE: Nationale Intensive Care Evaluatie; RR(R): Relative Risk (Reduction); RRT: Renal Replacement Therapy; SAE: Serious Adverse Event; SSC: Surviving Sepsis Campaign; SD: Standard Deviation; STK: Save The Kidney; VAP: Ventilator-associated Pneumonia

\section{Acknowledgements}

We thank lgor van der Weide for his support with the database.

\section{Authors' contributions}

Conceived and designed the study and analysis: JK, FK, $\mathrm{H}_{\mathrm{H}} \mathrm{JZ}$ and MM. Analyzed the data: JK and FK. Contributed to gathering the data: WD. Wrote the paper: JK, IH, RW, FK, EC, JZ and MM. The authors read and approved the final manuscript.

\section{Funding}

The project was supported by the research foundation of the Critical Care Department UMCG: Stichting Water en Lucht. Dr. van Meurs was supported by a Kolff grant of the Dutch Kidney Foundation (13OKJ35).

Availability of data and materials

Please contact the author for data requests.

Ethics approval and consent to participate

Informed consent was waived by the Institutional Review Board of the University Medical Center Groningen because the study had an observational design and all data were de-identified (METc 2013-174).

Consent for publication

Not applicable.

Competing interests

On behalf of all authors, the corresponding author states that there is no conflict of interest.

Received: 20 December 2019 Accepted: 19 August 2020

Published online: 03 September 2020

\section{References}

1. Gammelager $\mathrm{H}$, Christiansen $\mathrm{CF}$, Johansen MB, et al. One-year mortality among Danish intensive care patients with acute kidney injury: a cohort study. Crit Care. 2012;16:R124. https://doi.org/10.1186/cc11420.

2. Nisula S, Kaukonen KM, Vaara ST, et al. Incidence, risk factors and 90-day mortality of patients with acute kidney injury in Finnish intensive care units: the FINNAKI study. Intensive Care Med. 2013;39:420-8. https://doi.org/10. 1007/s00134-012-2796-5.

3. Bellomo R, Ronco C, Kellum JA, et al. Acute renal failure - definition, outcome measures, animal models, fluid therapy and information technology needs: the second international consensus conference of the acute dialysis quality initiative (ADQI) group. Crit Care. 2004;8:R204-12 https://doi.org/10.1186/cc2872.

4. Mehta RL, Kellum JA, Shah SV, et al. Acute kidney injury network: report of an initiative to improve outcomes in acute kidney injury. Crit Care. 2007;11: R31. https://doi.org/10.1186/cc5713.

5. Workgroup KAKI (2012) Kidney Disease: Improving Global Outcomes (KDIGO) Acute Kidney Injury Work Group. KDIGO clinical practice guideline 
for acute kidney injury. Kidney Int. 2012;2:1-138 http://www.kdigo.org/ clinical_practice_guidelines/pdf/KDIGOAKI Guideline.pdf.

6. Linder A, Fjell C, Levin A, et al. Small acute increases in serum creatinine are associated with decreased long term survival in the critically ill. Am J Respir Crit Care Med. 2014;189:1075-81. https://doi.org/10.1164/rccm.201311-20970C.

7. Vaara ST, Pettila V, Kaukonen KM, et al. The attributable mortality of acute kidney injury: a sequentially matched analysis*. Crit Care Med. 2014;42:87885. https://doi.org/10.1097/CCM.0000000000000045.

8. Ostermann $\mathrm{M}$, Chang RW. Acute kidney injury in the intensive care unit according to RIFLE. Crit Care Med. 2007;35:1837-43quiz 1852. https://doi. org/10.1097/01.CCM.0000277041.13090.0A

9. Joannidis M, Druml W, Forni LG, et al. Prevention of acute kidney injury and protection of renal function in the intensive care unit. Expert opinion of the working Group for Nephrology, ESICM. Intensive Care Med. 2010;36:392411. https://doi.org/10.1007/s00134-009-1678-y.

10. Haase N, Perner A, Hennings LI, et al. Hydroxyethyl starch 130/0.38-0.45 versus crystalloid or albumin in patients with sepsis: systematic review with meta-analysis and trial sequential analysis. BMJ. 2013;346:f839. https://doi. org/10.1136/bmj.f839.

11. Asfar P, Meziani F, Hamel JF, et al. High versus low blood-pressure target in patients with septic shock. N Engl J Med. 2014;370:1583-93. https://doi.org/ 10.1056/NEJMoa1312173.

12. Rivers E, Nguyen B, Havstad S, et al. Early goal-directed therapy in the treatment of severe sepsis and septic shock. N Engl J Med. 2001;345:136877. https://doi.org/10.1056/NEJMoa010307.

13. Dellinger RP, Lewy MM, Rhodes A, et al. Sunviving sepsis campaign: international guidelines for management of severe sepsis and septic shock, 2012. Intensive Care Med. 2013;39:165-228. https://doi.org/10.1007/s00134-012-2769-8.

14. Morris AC, Hay AW, Swann DG, et al. Reducing ventilator-associated pneumonia in intensive care: impact of implementing a care bundle. Crit Care Med. 2011;39:2218-24. https://doi.org/10.1097/CCM. 0b013e3182227d52.

15. Meersch M, Schmidt C, Hoffmeier A, et al. Prevention of cardiac surgeryassociated AKI by implementing the KDIGO guidelines in high risk patients identified by biomarkers: the PrevAKI randomized controlled trial. Intensive Care Med. 2017;43:1551-61. https://doi.org/10.1007/s00134-016-4670-3.

16. Kolhe NV, Staples D, Reilly T, et al. Impact of compliance with a care bundle on acute kidney injury outcomes: a prospective observational study. PLoS One. 2015;10:e0132279. https://doi.org/10.1371/journal.pone.0132279.

17. Vogelzang M, Zijlstra F, Nijsten MW. Design and implementation of GRIP: a computerized glucose control system at a surgical intensive care unit. BMC Med Inform Decis Mak. 2005;5:38. https://doi.org/10.1186/1472-6947-5-38 [pii].

18. Colpaert K, Hoste EA, Steurbaut K, et al. Impact of real-time electronic alerting of acute kidney injury on therapeutic intervention and progression of RIFLE class. Crit Care Med. 2012;40(4):1164.

19. Udy AA, Roberts JA, Lipman J. Clinical implications of antibiotic pharmacokinetic principles in the critically ill. Intensive Care Med. 2013;39: 2070-82. https://doi.org/10.1007/s00134-013-3088-4.

\section{Publisher's Note}

Springer Nature remains neutral with regard to jurisdictional claims in published maps and institutional affiliations.

Ready to submit your research? Choose BMC and benefit from:
- fast, convenient online submission
- thorough peer review by experienced researchers in your field
- rapid publication on acceptance
- support for research data, including large and complex data types
- gold Open Access which fosters wider collaboration and increased citations
- maximum visibility for your research: over 100M website views per year
At BMC, research is always in progress.
Learn more biomedcentral.com/submissions

